\title{
Vorwort.
}

Das vorliegende Buch - eine freie deutsche Bearbeitung und Erweiterung von B. A. Behrend "The induction motor" verfolgt den $Z$ weck, Studierenden und in der Praxis stehenden Ingenieuren, die ein Interesse daran baben, das Wesen der Induktionsmotoren kennen zu lernen, aber nicht die Zeit gewinnen können, das far sie Brauchbare aus der Hochflut der einschlägigen Litteratur zu sammeln, ein Kompendium zu geben, in dem der heutige Stand der Theorie und Praxis der Induktionsmotoren erschöpfend behandelt ist.

Um dieses Ziel zu erreichen und damit eine bestehende Lucke in der Fachlitteratur auszufullen, war der Herausgeber gezwungen, gewisse Rücksichten auf den Umfang der Schrift zu nehmen, und infolgedessen soviel Kenntnisse auf dem Gebiete der Wechselstrom-Technik vorauszusetzen, als vom akademisch gebildeten Ingenieur heute verlangt werden kann. Theoretische Ableitungen wurden daber nur soweit aufgenommen, als sie für das Verstăndnis und die praktische Anwendung der speciellen Probleme auf dem hier behandelten Gebiet unerlässlich sind.

Auf Einheitlichkeit der Darstellung ist Wert gelegt worden, erreicht konnte sie auch dort werden, wo auf vorhandene Arbeiten zurückgegriffen wurde, wobei natürlich die Bezeichnungen teilweise entsprechend geändert werden mussten.

Die konstruktive Behandlung des mechanischen Teiles ist, soweit sie nicht durch den allgemeinen Maschinenbau erledigt wird, beracksichtigt worden. Die Anwendungen lassen sich an der Darstellung typischer und extremer Falle am 
natürlichsten erlăutern, und es sind deshalb solche an Hand praktischer Ausführungen als Beispiele aufgenommen worden; dagegen wurde auf die Reproduktion von Reklamebildern verzichtet, ebenso auf "Rezept-Sammlungen“, welche die Berechnung eines Motors "ohne Weiteres“ gestatten.

Bei dieser Gelegenheit sage ich der Elektrizitäts-ActienGesellschaft vorm. Lahmeyer \& Co. in Frankfurt a. M., der Aktien-Gesellschaft Siemens \& Halske und insbesondere Herrn Ingenieur Alfred Kolben in Firma Elektrizităts-AktienGesellschaft vorm. Kolben \& Co., Prag, für die liebenswärdige Ueberlassung von konstruktivem Material meinen verbindlichsten Dank.

Graphische Darstellungen sind durchweg bevorzugt worden, da der Techniker mit gutem Recht von allen Begriffen, mit denen er zu operieren hat, verlangt, dass sie greifbar und sinnlich wahrnehmbar erscheinen.

Aus diesem Grunde wurde die symbolische Rechnung mit komplexen Grössen - eine zwar geistreiche aber doch künstliche Schreibweise, welche die graphischen Methoden nicht ersetzen kann - weggelassen. Sie bietet dem Erfahrenen zwar den Vorzug, dass sie schwierigere Rechnungen vereinfacht, für den Anfãnger aber ist sie wie ein Tunnel, der dem Fahrenden die Möglichkeit abschneidet, sich des Weges bewusst $\mathrm{zu}$ werden, auf dem er sich befindet.

Charlottenburg, im Februar 1903.

\section{Dr. Paul Berkitz.}

